\title{
Retroperitoneal Schwannoma mimicking a Large Adrenal Incidentaloma: Case Report and Review of Literature
}

\author{
${ }^{1}$ Shafiq Rahman Abdul Gaffoor Shabiyullah, ${ }^{2}$ Reyaz M Singaporewalla, ${ }^{3}$ Anil Dinkar Rao, ${ }^{4}$ Vijayadwaja Desai
}

\begin{abstract}
Schwannomas are rare tumors originating from Schwann cells of peripheral nerve sheath (neurilemma) of the ectoderm. They are also referred to as neurilemmomas and are generally benign, solitary tumors arising from cranial and peripheral nerves. Rarely, they arise from the retroperitoneum and adrenal medulla. Retroperitoneal schwannomas constitute about 0.5 to $5 \%$ of all schwannomas and of which most of them are benign in nature. Most patients are asymptomatic or have vague abdominal symptoms. On imaging, they are often mistaken for adrenal tumors due to their location and preoperative diagnosis is often difficult. Magnetic resonance imaging (MRI) is the investigation of choice to demonstrate these lesions and its relation to other structures. Total excision is recommended as malignancy, although rare cannot be ruled out preoperatively. We present a case of a large retroperitoneal schwannoma that mimicked an adrenal incidentaloma and was excised laparoscopically. The imaging characteristics and management strategy are discussed.
\end{abstract}

Keywords: Retroperitoneal schwannoma, Adrenal incidentaloma, Laparoscopic adrenalectomy.

How to cite this article: Shabiyullah SRAG, Singaporewalla $\mathrm{RM}$, Rao AD, Desai V. Retroperitoneal Schwannoma mimicking a Large Adrenal Incidentaloma: Case Report and Review of Literature. World J Endoc Surg 2014;6(2):81-84.

\section{Source of support: Nil}

Conflict of interest: None

\section{INTRODUCTION}

Retroperitoneal schwannomas constitute about 0.5 to $5 \%$ of all schwannomas. They are usually benign, solitary lesions arising from the adrenal medulla. Most patients are asymptomatic or have vague abdominal symptoms. ${ }^{1}$ On imaging, they are often mistaken for adrenal tumors due to their location and preoperative diagnosis is often difficult. Diagnosis is often made after surgical excision using immunohistochemistry techniques. We present a case of a large retroperitoneal schwannoma that mimicked an adrenal

\footnotetext{
${ }^{1}$ Medical Officer, ${ }^{2,4}$ Consultant, ${ }^{3}$ Registrar

${ }^{1-3}$ Department of General Surgery, Khoo Teck Puat Hospital Yishun Central, Singapore

${ }^{4}$ Department of Pathology, Khoo Teck Puat Hospital, Singapore

Corresponding Author: Reyaz M Singaporewalla, Consultant Department of General Surgery, Khoo Teck Puat Hospital 90 Yishun Central, Singapore, Phone: 6565558000, e-mail: reyazm@yahoo.com
}

incidentaloma on imaging and was excised laparoscopically. We describe the imaging characteristics of these lesions and review the literature.

\section{CASE REPORT}

A 42-year-old Chinese female presented with a 1 month history of abdominal discomfort. Abdominal examination was unremarkable. She denied any symptoms of anxiety, palpitations or headaches. Ultrasound abdomen suggested a right adrenal mass measuring $4.3 \mathrm{~cm}$ and an adjacent $3 \mathrm{~cm}$ hypoechoic lesion in the right adrenal gland. Computed tomography (CT) scan of adrenal glands reported a dumbbell shaped mass in the region of the right adrenal gland, with two large lobulations, anteriorly $(6.3 \mathrm{~cm}$ diameter $)$ and posteriorly ( $2.5 \mathrm{~cm}$ diameter). The mass showed precontrast and postcontrast attenuation values of $37 \mathrm{HU}$ and $68 \mathrm{HU}$ respectively with a delayed postcontrast attenuation of $85 \mathrm{HU}$. There was no washout in the delayed phase to suggest an adenoma. There was mass effect with narrowing of the inferior vena cava (IVC) and displacement of the right lobe of the liver laterally (Figs 1A and B). There was no fat density within to suggest myelolipoma. The left adrenal gland was noted to be normal.

Routine hormonal investigations for an adrenal mass such serum renin, aldosterone and cortisol were unremarkable. Twenty-four hours urine cortisol and pheochromocytoma screen were within normal range. The workup essentially showed a large nonfunctioning $>6 \mathrm{~cm}$ adrenal solid mass. The patient was advised surgery in view of the large size of the incidentaloma and its mass effect on the IVC. She underwent a laparoscopic right adrenalectomy, with an uneventful outcome and was discharged after on the second day.

Intraoperatively, the mass was found to be well circumscribed and free from the surrounding structures. It was impossible to separate the mass from the right adrenal gland and an en-bloc resection was carried out to prevent tumor capsular breach (Figs 2A and B).

Histology of the resected lesion showed a well-defined solid mass with areas of central cystic degeneration. It measured $90 \times 45 \times 35 \mathrm{~mm}$ and weighed 60 gm. Microscopy showed a nodular encapsulated spindle cell lesion growing in fascicles. No significant cellular atypia or mitotic activity 
was noted. Nerve trunks and ganglion cells were seen in the periphery of the lesion. Immunohistochemistry showed that the tumor cells were strongly and diffusely positive for $\mathrm{S} 100$ and only focally/variably positive for smooth muscle actin and Bcl-2. They were negative for CK 7, CD 117, MCK and Melan A. Ki-67 proliferative index was approximately $1 \%$. The final impression was a benign schwannoma with normal adjacent adrenal gland (Figs $3 \mathrm{~A}$ to $\mathrm{C}$ ).

\section{DISCUSSION}

Schwannomas are rare tumors originating from the Schwann cells of the peripheral nerve sheath (neurilemma) of the ectoderm. They are also referred to as neurilemmomas. These are generally benign, solitary tumors that arise mainly from cranial and peripheral nerves. ${ }^{2}$ Rarely, they can arise from the retroperitoneum and adrenal medulla. It has been theorized that schwannomas in this region originate from Schwann cells that insulate the nerve fibers innervating the adrenal medulla. ${ }^{3}$ Only around 0.5 to $5 \%$ of benign schwannomas are found in the retroperitoneal region. ${ }^{1}$ Preoperative diagnosis of a schwannoma of the adrenal gland is often incidental, because majority are deeply situated tumors which are usually present for long periods of time. In large tumors, patients may complain of vague abdominal discomfort due to mass effect, which is the commonest symptom. ${ }^{3}$ Rarely atypical presentations can occur and include dysesthesia, headache, secondary hypertension, flank pain, hematuria or recurrent renal colic. ${ }^{1}$ Laboratory investigations for schwannoma are unremarkable and do not contribute to diagnosis. ${ }^{4}$

There are different imaging modalities that may aid in the differential diagnosis of retroperitoneal schwannomas, such as ultrasonography, CT-scan and MRI. ${ }^{1,5}$ The various lesions that can occur in these regions can be an adenoma of the adrenal gland, pheochromocytoma, myelolipoma, metastasis, neurofibroma, paraganglioma, lipoma and malignant fibrous histiocytoma. ${ }^{1}$ Schindler et $\mathrm{al}^{6}$ suggested that MRI is the imaging modality of choice for soft-tissue lesions as it allows better demonstration of origin, vascular architecture and relation to surrounding structures. Hughes et $\mathrm{al}^{7}$ have described the MRI characteristics of retroperitoneal schwanommas. They typically appear as a wellcircumscribed homogenous mass with slight enhancement
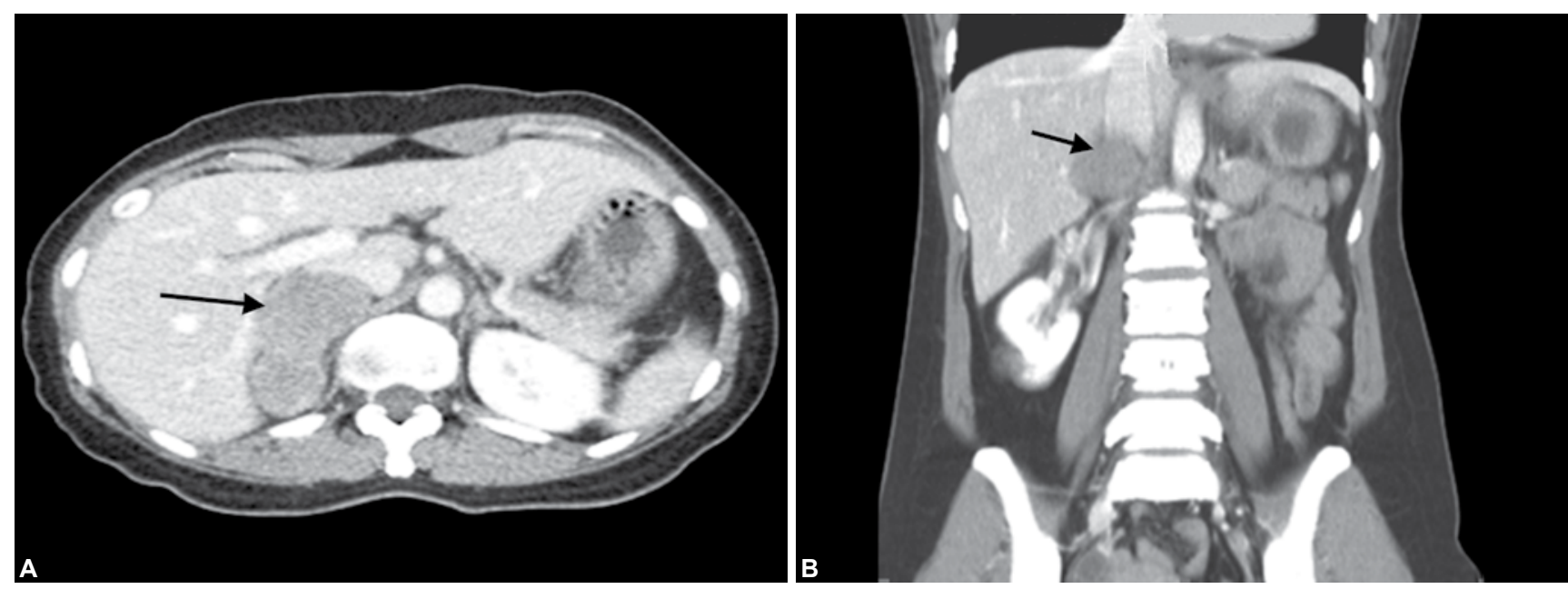

Figs $1 \mathrm{~A}$ and B: Axial and coronal CT images showing a dumb-bell shaped lesion in the region of the right adrenal with compression of inferior vena cava (black arrow)
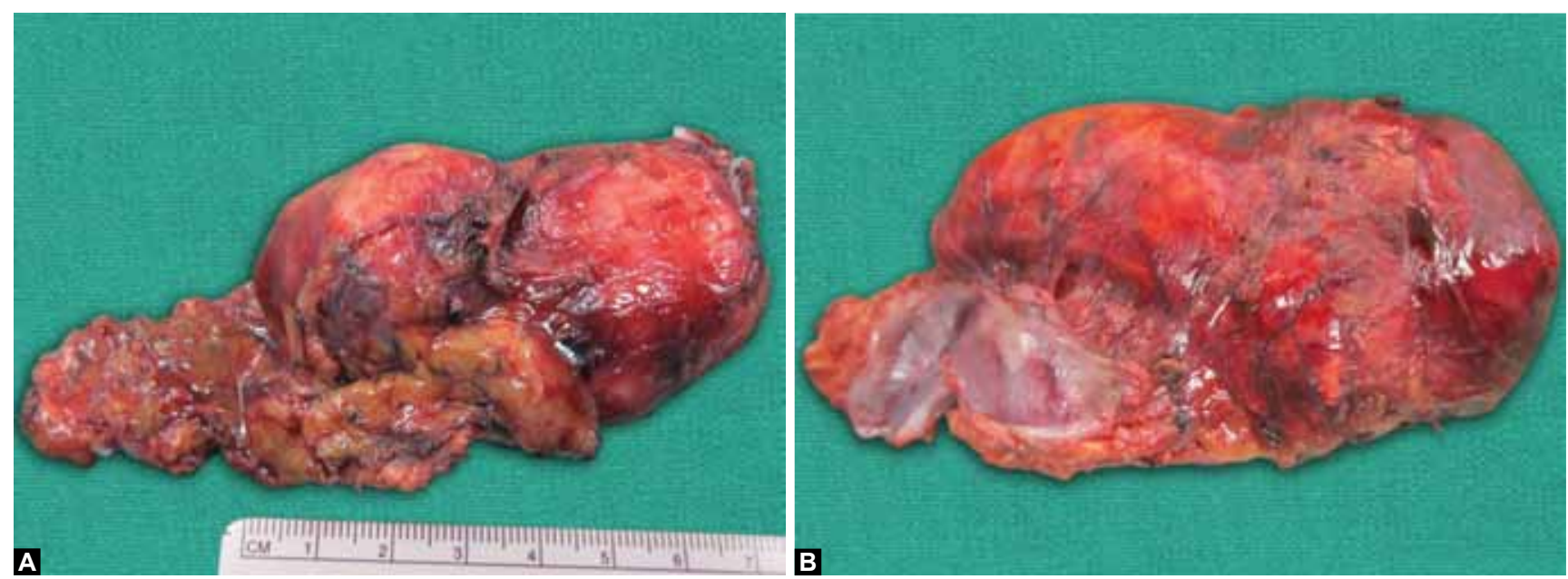

Figs 2A and B: Posterior and anterior view of resected specimen 
after administration of contrast. One specific finding is low signal intensity on T1-weighted images and high signal intensity on T2-weighted images. The outphase images show a signal loss. Albeit nonspecific, these findings are reported to be present in $57 \%$ of cases. ${ }^{1}$ We did not perform a preoperative MRI in our patient as the possibility of a schwannoma was not entertained preoperatively by our radiologist and secondly because it would not change our management for surgical excision in view of its size.

Schwannomas are almost benign in nature and in rare occurrence it undergoes malignant transformation. Malignant schwannomas are aggressive tumors that act as high grade sarcomas and usually associated with Von Recklinghausen's disease. Benign schwannomas have a low rate of recurrence and a very good prognosis whereas malignant schwannomas have a higher rate of recurrence and poor prognosis. They are also insensitive to chemotherapy and radiotherapy. ${ }^{2,5,8,9}$

In general, preoperative biopsies are not recommended for these lesions by most authors. However, Schindler et $\mathrm{al}^{6}$ regarded needle biopsy as the diagnostic gold standard and
Hughes et $\mathrm{al}^{7}$ used core biopsy combined with imaging features to decide on conservative nonsurgical management. In our case, we did not perform a preoperative biopsy as it would not change the management plan for surgical excision. Imaging modalities cannot provide a definite diagnosis and surgical resection of such tumors, especially large ones is necessary to make a diagnosis and differentiate it from other soft-tissue tumors and rule out malignancy. ${ }^{1}$ However, according to Ohta et $\mathrm{al}^{10} \mathrm{MRI}$ is a preferred imaging modality over CT scan in all suspected perinephric and retroperitoneal schwannomas. CT-scan has its limitations in the providing an image diagnosis due to its lower soft-tissue resolution as compared to MRI, and in certain situations can mislead the diagnosis as in our case. CT images do not enhance stroma heterogeneities, a main characteristic in ancient Schawannoma, compared to MRI. ${ }^{6}$ The features highly suggestive of a schwannoma on MRI include (a) heterogenous contrast enhancement owing to cystic and hemorrhagic changes, (b) calcification, (c) Iso or slightly hyperintense signal on T1-weighted images and high signal intensity on $\mathrm{T} 2$-weighted images. ${ }^{10}$ In addition, to the standard $\mathrm{T} 1$ and T2-images,
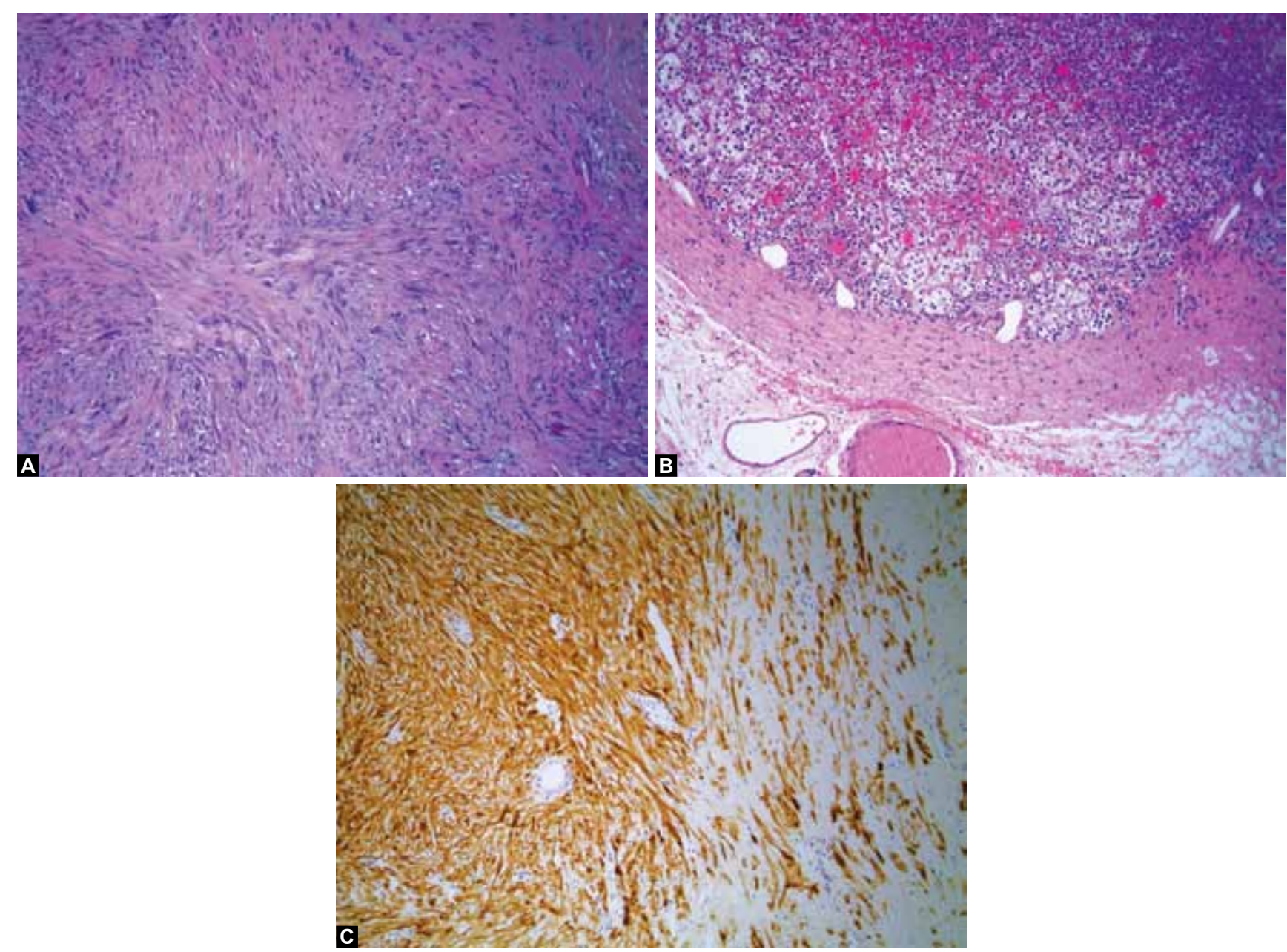

Figs $3 A$ to $C:(A)$ and $(B)$ : Microscopic views showing benign schwannoma cells along with alternating Antoni $A$ and Antoni $B$ patterns with fibrinoid degeneration, thrombosis and hyalinization (H\&E staining, magnification $\times 100)$, (C): Immunohistochemical stain positive for S-100 protein 
Schindler et $\mathrm{al}^{6}$ have recommended the acquisition of a fat suppression sequence (STIR) on which the schwannoma will maintain its high signal allowing for delineation from pure lipomatous tumors. The understanding regarding surgical therapy is complete extirpation. In our case, the mass was closely adhered to the adrenal gland and no plane of separation was present between the two structures. Attempting to separate the adrenal gland from the schwannoma intra-operative is also not advisable due to risk of capsule rupture. Literature reports suggest that a schwannoma arising from adrenal medulla is very rare with only 3 reports thus far. ${ }^{1}$

Histology with immunohistochemistry provides the confirmed diagnosis of a schwannoma with two distinct subtypes of areas (Antoni type A and B areas). Microscopy of Antoni A areas show short spindle cells with long conical nucleus and scant cytoplasm forming a spiral structure. Antoni B areas show inflammatory cells scattered within a loose matrix. ${ }^{1}$ Other findings strongly supporting the diagnosis are a strong positive staining for S-100 protein in the cytoplasm of the tumor cells and collagen IV in immunohistochemical analysis with absence of reactivity for keratin, actin and CD34.

The authors consider a retroperitoneal schwannoma mimicking an adrenal incidentaloma to be no different from a schwannoma arising from the adrenal medulla, with preoperative confirmative diagnosis not possible in both cases. Baseline hormonal studies to rule out adrenal hyperfunction are necessary when the lesion cannot be separated from the normal adjacent adrenal gland. The guidelines for surgical excision should therefore be the same as for nonfunctioning adrenal incidentalomas based on their size and imaging characteristics. Baseline hormonal studies to rule out adrenal hyperfunction are necessary when the lesion cannot be separated from the normal adjacent adrenal gland. Retroperitoneal schwannomas mimicking adrenal lesions have been described in the literature ${ }^{2}$ and laparoscopic adrenalectomy has been advocated as the standard treatment based on the size. The prognosis after excision of a benign schwannoma is good although malignant transformation has been reported. Follow-up with imaging is suggested after surgical excision of the lesion. ${ }^{11}$

\section{CONCLUSION}

Retroperitoneal schwannomas are often difficult to differentiate from adrenal incidentalomas and a high index of suspicion is required for preoperative diagnosis. MRI scans can help in differentiating these lesions and follow-up of small tumors. For large $(>6 \mathrm{~cm})$ tumors standard recommendation is for complete surgical resection in the form of laparoscopic adrenalectomy after completing the baseline hormonal evaluation.

\section{REFERENCES}

1. Konstantinos TG, Dimitrios T, Panagiotis KB, Nikolaos MV, Ioannis F, Andreas M, Geogrios Z. Laparoscopic resection of an adrenal schwannoma. JSLS 2012;16:663-637.

2. Sharma SK, Koleski FC, Husain AN, Albala DM, Turk TM. Retroperitoneal schwannoma mimicking an adrenal lesion: case report. World J Urol 2002;20:232-233.

3. Korets R, Berkenblit R, Ghavamian R. Incidentally discovered adrenal schwannoma: case report. JSLS 2007;11:113-115.

4. Inokuchi T, Takiuchi H, Moriwaki Y, Ka T, Takahashi S, Tsutsumi Z, Shima H, Hirota S, Yamamoto T. Retroperitoneal ancient schwannoma presenting as an adrenal incidentaloma: CT and MR findings. Magnetic Resonance Imaging 2006;24:1389-1393.

5. Li Q, Gao C, Juzi JT, Hao X. Analaysis of 82 cases of Retroperitoneal Schwannoma. ANZ J Surg 2007;77:237-240.

6. Schindler OS, Dixon JH. Retroperitoneal giant schwannomas: report on two cases and review of the literature. J Orthopaedic Surgery 2002;10:77-84.

7. Hughes MJ, Thomas JM, Fisher C, Moskovic EC. Imaging features of retroperitoneal and pelvic schwannomas. Clinical Radiol 2005;60:886-893.

8. Goh BKP, Tan YM, Chung YFA, Chow PKH, Ooi LPJ, Wong WK. Retroperitoneal Schwannoma. Am J Surg 2006;192:14-18.

9. Pinto D, Person OK, Cho M, Zundel N, Szomstein S, Rosenthal RJ. Laparoscopic resection of a retroperitoneal degenerative schwannoma: a case report and review of the literature. Surg Laparos Endosc Percutan Tech 2008;18:121-123.

10. Ohta I, Lin PH, Rau CL, Wang KC. Evaluation of perinephric, retroperitoneal schwannomas: case report and review of the literature. South Med J 2007;100:80-82.

11. Cury J, Ferreira C, Srougi M. Retroperitoneal schwannoma: case series and literature review. CLINICS 2007;62:359-362. 